\title{
Designing Ubiquitous Computing Systems for Sports Equipment
}

\author{
Matthias Kranz, Wolfgang Spiessl, Albrecht Schmidt \\ Embedded Interaction Research Group, University of Munich \\ Amalienstrasse 17, 80333 Munich, Germany \\ \{matthias, wolfgang, albrecht\}@ hcilab.org
}

\begin{abstract}
In this paper, we report on a user-centered, iterative design process for augmenting sports equipment with ubiquitous computing technology. In several design iterations, a fully working system for training and physiotherapy has been developed and deployed using hard-and software components of ubiquitous computing technology. We report on the design and development process that led to this system. Based on our experience we generalize the specific processes to general ubicomp systems. The validity of our approach has been verified by a larger user study.
\end{abstract}

\section{Introduction}

Designing ubiquitous computing systems for end users is a challenging task. The challenges for the system engineering process are inherently different from those for systems operating in a laboratory setting operated by software engineers and researchers. End users, especially those without a technical background, have a different focus on computing technology and interaction devices. They might have problems clearly state requirements or when interviewed they might report what they are supposed to do but not how they actually achieve it with the current systems. This has often been reported in the literature for software engineering. With physical or tangible, explicit or implicit interaction commonly found in ubiquitous computing systems, users are even less able to clearly state their requirements. This is especially true if novel (to the user) forms of interaction are involved. New and adapted processes are needed to elicit requirements and engineer systems.

In this paper we report on a a successfully developed and deployed system in the field of sports. We present the design and development process and provide reasoning for each step. We highlight aspects that are generally applicable for a wide range of interactive ubiquitous computing systems designated to be used by end users in a real scenario.

Ubiquitous computing technologies in these fields use small embedded devices with sensor input to create devices that allow totally new applications and experiences [3, 9].
Our system [16] consists of several pieces of sports equipment augmented with sensors, communication and processing. By recording and monitoring exercises and by providing audio-visual feedback, new experiences can be created. The technology enables users to see how well they perform their exercises and helps to correct and optimize their training. Monitoring and visualizing advances inform and motivate users. Additionally, the automated continuous feedback eases the task of physiotherapists and coaches as it reduces human supervision and hence also the cost. The technical details of the system can be found in [12].

We explicitly chose a scenario where a relatively simple technology like a wireless sensor node with acceleration sensors can be used to create a meaningful end user system that can be deployed and maintained by ordinary people and users. This allows us to look in more detail in the challenges for the design and development process.

The contributions of the paper are as follows. First, we report on the positive experiences with technology probes and an extended user-centered design process. We show how a requirement analysis was done and report on the iterations to actually build the final system. We demonstrate that knowledge transfer within the interdisciplinary project team is highly necessary and can be achieved. We also report on how the process can be generalized for other ubiquitous computing projects.Second, we briefly describe technological aspects of the deployed system with regard to the overall integration in the existing infrastructure and explain the lessons we learned in this process.Third, we report on a two-weeks continuous user study with 47 participants doing 100 training sessions in a large sports school. The study confirmed that novel technologies, if they focus on the users' needs, can achieve great acceptance with users and operators and that new technology needs to reach a high level of functionality, beyond the prototyping stage, to be meaningfully assessed.

\section{Related Work}

Sensor support for competitive athletes is very common, for example in football or in skiing [14] or Taekwondo [2] used for independent judgment of the athlete's performance. 
The technology used has to be accepted by all participants and should work in the background, which we also found to be a crucial issue. Issues related to social acceptance factors are important and have to be taken into account as early as possible in the design process, otherwise the system will always be problematic, as discussed for several deployed systems in [14].However, it needs to be mentioned that in professional sports practice the acceptance for technology (even if not very usable) is high when it offers a completive advantage. The benefits of a personal fitness and health monitoring device, the Personal Wellness Coach, have been discussed in [1].

The positive effects of electronically augmented sports equipment, which we also experienced in our project, have been shown in the Thera-Network in [10]. How computeraugmented sports devices increase the motivation of especially young children to do sportive exercises is subject to the Fizzees project [15]. The sensor system is used only as vehicle to achieve the overall goal of improved fitness.

The electronics used and the architecture for communication sensor data to Flash developed in the SensorVirrig Project [6] helped us during system development.

Technology probes [4, 8], as used by us during the development cycles, are an effecive method for assessing the needs and wishes of end-users in real-world settings and to develop a common understanding on the potentials of the technology. They also are able to foster the users' creativity to come up with new ideas.

\section{The Design and Development Process}

In the UbiFitness project [12, 16], we explored options for the development of ubiquitous computing systems for sports. The overall philosophy was to focus on stakeholders' needs while developing the best possible technical solution. To achieve this we consequently followed a user centered design process that was extended by the use of technology previews and technology probes. The development was done in several iterations, creating prototypes of different fidelity. These have all been deployed and tested with users in the real use environment. During the process a multi-disciplinary team of physiotherapists, sport coaches and computer scientists were involved.

We used different methods and tools during the iterative design process. The objective was to build a system suited and customized perfectly to the respective needs of the users and based on the latest technology available. The advances with regard to the technology were rather on the system architecture side than with the sensing system developed. The technology components were used as vehicle to achieve the goal - a working and deployable ubiquitous computing prototype. The overhead necessary for our extended design process is significant but results justify its employment. At the end of each step, we generalize our findings.
In particular, the following elements are of interest and proved to be important for the success:

\subsection{Stakeholder Identification}

It has been a basic principle of our approach that during the whole process the interests of user and stakeholders drive the development. From the plentitude of definitions (see Sharp et al. [19] for an overview) for stakeholder, we find that the definitions most appropriate for ubiquitous computing is given by Friedman et al. [5] which is used by Scholtz and Consolvo for their evaluation guidelines [18].

"Direct stakeholders refer to parties - individuals or organizations - who interact directly with [the system] or its output. Indirect stakeholders refer to all other parties who are affected by the use of the system."

Neglecting to identify and involve all stakeholders, in the worst case, invalidates all results and the system based on them. This applies to all ubicomp systems involving end users, researchers probably will be able to deal with imperfect system, they do so every day.

We identified all potential stakeholders before any development to make sure the system can be evaluated according to the special needs of ubiquitous computing systems, as discussed by Scholtz et. al [18].

\subsection{Technology Developers Learn About the Domain in Depth}

Even though the development took place in a multidisciplinary team, it was invaluable that members from each discipline had an in-depth understanding of the other fields. To understand the application domain one of the computer scientists regularly attended a sports school as 'customer' and 'user'. This was done before the start of any development in the project for a period of more than three months doing regular sessions. This gave the researchers insight in how people are taught to do the exercises, how reporting and error-correction is done. This input was essential for the technology developers to gain an understanding of the application domain and the work processes. Gaining knowledge in the domain helped to chose an initial set of prototyping materials, which we later report on.

In general, the period of time depends on many environmental factors. It should be long enough to allow the researcher to "move and act naturally" in all settings.

In our case study after that period, unstructured interviews with both trainers and trainees were conducted looking for problem domains with possible improvement. These interviews influenced many (small but crucial) decisions that had to be taken in the course of a project. The documentation and discussion of these results provided 
a common basis for the development process. This was especially helpful for the "expectation management" for end users - any system that fails to meet the expectations of users will suffer acceptance problems. Users may initially not be aware of what is possible with what effort. Joint discussions helped to build a system acceptable for all stakeholders. This also helps to create a feeling of ownership among the end users. This method of interaction design should be applied in any interactive ubiquitous computing system designed to be used by "normal" people. This, again, may be different for research environments.

\subsection{Educating the User: Technology Previews and Technology Probes}

To communicate the potential of available technology, several demonstration systems were used. These technology previews - either quick 'hacks' or systems developed in previous projects - outlined basic functionality, such as real-time data capture with sensors, wireless data transmission, and real-time visualization. Placing the technology in the context of the application domain inspired non-technical members of the team and showed restrictions (data sensing and communication, etc.) at the same time.

Giving some technology parts, even if not handled perfectly, to a domain expert proved to be very valuable, as in further discussions they referred to what they knew was possible. We did not include end users in this process because to understand a specific system after only seeing a crude technology preview requires an in-depth and rather abstract understanding of the processes and goals. Our experience showed that many users at school lack such knowledge. For end users, even prototypes need a certain level of maturity. This is necessary to allow them to think aloud about the possibilities and not distract them with the shortcomings of the specific prototype. The maturity here refers to the stability and functionality of the system as well as its design. Regarding the latter, it is always easier for a user to state what he dislikes about a current instance of a system than how he would like it to be. This is especially important for the look and feel and the affordances of physical user interfaces, e.g. tangible user interfaces.

In the following subsections we present the prototyping methodologies and tools we used for educating the users and for exploring the design space.

\subsection{Paper Prototypes and Mock-Ups}

Paper prototypes and mock-ups were used as communication tools in early phases of the development. Using paper prototypes proved to be an efficient way for informal system specification and to document requirements. As all participants could equally influence the appearance and 'function- ality' of the prototypes, this methods allowed a truly multidisciplinary development. Not surprisingly, this worked especially well for issues related to visualization (hardware and software) and documentation. It was interesting to see, however, that these methods have limits when it comes to integration and (real) time functionality and flow of information within the system. Here a great level of abstraction is required to see how such prototypes work. It seems that this is easier for technologists than for the other people involved. For this, other more physical, approaches from information visualization seem more promising, e.g. "physically" moving the data along the system.

\subsection{Rapid Prototyping Support}

Throughout the project we developed functional prototypes with different fidelity. Depending on the investigated question and the target audience, these prototypes ranged from simple demonstrators highlighting a single issue to the final fully functional prototypical system that is integrated into the existing infrastructure. Nevertheless, we learned that providing real functionality is essential to assess experience, especially if it is novel. Also, it is impossible to tackle more than one or two specific issues at once.

Iterative development is the only way to address this problem. For demonstrators and simple prototypes we used Flash. Graphical elements here can be defined and changed very quickly which allowed the rapid prototyping of the user interface and the exploration of different options for the visualization. Toolkits as e.g. the Flash prototyping toolkit for the Pin \& Play platform are invaluable tools for this. Also, middlewares that allow for separate hardware and software development by "faking" event like Papier-Mâché [11] are helpful. Making use of existing toolkits, middlewares and hardware platforms speeds up the development process. This allows the restricted amount of time for every development project to be used more effectivley. Running out of time, especially in normal software engineering projects, is often the reason for not conducting e.g. usability tests. For ubiquitous computing systems where everyday objects and their affordances and the environment "become" the user interface this is of great importance.

\subsection{Functional Prototypes - Troughout the Development}

Low level functionality (e.g. acquisition of sensor data) was developed early in the project and remained basically the same throughout the whole development process. It was very valuable to have developed these components early in the project and to provide an easy to access interface to have the data available for prototyping. We used an off-the-shelf wireless sensor network platform for data acquisition and sockets for communication. 
Restriction, modification and extension of off-the-shelf components as proposed by Kranz and Schmidt [13] is one possible way to quickly obtain suitable prototypes for interactive ubiquitous computing systems. By having functional prototypes, the experience and usage becomes very realistic and people using the system come across more issues than when just thinking or discussing about it. Issues like privacy surfaced only when people used the system, not when they just reflected on the potential use of a system. It was also very valuable for the motivation of all involved parties to have always a working prototype available for discussion. Accepted versions were kept also for documentation.

\subsection{Iterative Development and Fast Development Cycles}

At the early iterations the system was built on standard hardware, hardly integrated with the target environment and very limited in scope and functionality, but functional. In later stages specific hardware was developed, integrated with the existing infrastructure, and with an extended functionality. Even though functionality was limited at the beginning, having a functional system from the first step on to iterate over was important.

Ease of use, for all people dealing with the system, was a central issue during all iterations. Some user features, that increased the usability significantly, required the integration with the infrastructure. Therefore there was often a question of priorities - what features to include at what effort. Here, our target was to choose the parts that allowed the best improvement with regard to the user experience from step to step.

Having quick development cycles was important as in ubiquitous computing systems many components have an impact on the experiences created with the overall system. Packet-loss and communication delays in the wireless sensor network used to acquire sensor data can be tackled in multiple ways. In our example, designing a visualization that is robust against a certain amount of data loss was one solution which came naturally in the iterative design process as the iterations in the visualization were quicker than in the development of network protocols.

Each step in the iterative development was usually comprised of the following parts: creation of an idea for a design or improvement of a design, specification of the requirements and assessment of the technical feasibility, feedback from the whole project team on the specification, prototype or implementation, demonstration or deployment in the real use environment, and evaluation. This approach can be compared to agile software development in standard software engineering.

In extreme programming, a corresponding approach would be the small releases practice. Start with the smallest useful feature set. Release early and often, adding a few features each time. In general, methods from XP and agile software development proved to be very successful during all of our ubiquitous computing projects. Standard processes seem to be not flexible enough. Recent work on human-centered software design tends to incorporate the necessary methods more though.

\section{Case Study: Therapy Top}

After an initial study on potential candidates for a novel augmented piece of sports equipment, the therapy top was chosen. The detailed technological description of the developed system along with the medical background have been described in [12, 16]. Therefore we here only focus on the aspects of the design and development process.

The stakeholders involved in the project were users who did their training on the therapy tops, the coaches and the IT administrators of the training and management system. These minimum functional requirements to a meaningful deployed system were defined together by domain experts in medicine, rehabilitation, coaches, users and technologists. Only by several joint sessions of all the involved stakeholders, we were able to derive this list.

- capture orientation and tilt of one or two therapy tops

- wirless data transmission as cable would be hindering

- data sampling rate of about $50 \mathrm{~Hz}$

- long-term data storage (in XML)

- tilt angle calculation

- angular data accuracy of at least 3 degrees

- audio-visual training support using pre-recorded videos

- near-realtime training visualization

- authentication support and per-user profiles

- integration with the existing system

- exercise definition and assignment with a graphical editor

\subsection{Technology Preview, Mock-Ups and Paper Prototypes}

Before any technical implementation, the physiotherapists and coaches were shown a sensor data capturing device. We used the sensor technology already developed for the SensorVirrig [7, 17] and the race game application developed for it. A small program visualized the orientation and state of a physical object. The simultaneous demonstration of sensor device and visualizing application helped to create a deeper technological understanding.

Along with this hardware, several paper prototypes of potential applications related to the therapy top were 
discussed. These mock-ups included drawings of a virtual therapy top and angular and tilt values and a game which could be controlled by a microcontroller. It was explained that if this was included in the therapy top, children could playfully do their exercises while actually performing their training.

Presenting the people involved in the design team with a technology preview was essential to make them understand what is technologically feasible. Combining an understanding of technology and domain-specific background in sports and rehabilitation, we could determine the key parameters that would be necessary for a meaningful system.

We used Flash for visualizing the orientation of the therapy top during our initial meetings as it allowed us to quickly prototype user interfaces.

The $\mathrm{C}$ program for sensor data acquisition remained basically the same throughout the whole development process. The iterations were concentrated on a better bandwidth utilization of the available RF slots.

The first version of the graphical user interface required manual input of the user's name and exercise parameters (e.g. number of repetitions, start direction (e.g. forward/backward) and minimum and maximum tilt angles). This version used a standard laptop (no touch screen) as well as mouse and keyboard for data entry.

The initial visualization used one circle for each 'circle' or 'tilt' made with the therapy top, displaying small points at each angle where sensor data were captured, independent of the exercise done at that moment. An even distribution of the data points showed that the exercise was smoothly done in the case of the circling exercise.

\subsection{Development Cycles}

This visualization was discussed in a scheduled meeting of the stakeholders. It was found that displaying a large number of circles and data points would be distracting for the user. Therefore the visualization was changed to display only the last circle. In the last iteration, the circle was partitioned into sectors of 30 degrees. These were colored if any data was received in this sector. It is possible that no data at all is received if e.g. the user simply does not enter this area or if RF packet loss is too high. The color of the sector is either red, yellow, green or grey. The whole data for a segment is analyzed before coloring takes place. Green here means that the user a) entered this sector and b) kept within the defined parameters for the exercise such as the maximum and minimum tilt angle. Yellow means that the user kept most of the time within the defined boundaries. Red finally means a substandard performance in this sector. The initially grey sector stays grey if no data is received at all. This variant allows for easy judgement of the current exercise quality with a minimum of cognitive load and attention required by the user. For this reason we did not represent the current tilt angle as length of the arrow (a longer arrow representing a larger angle) either. The current 'circle' is updated in nearreal time to allow for a fast feedback. Thus, the trainee is able to determine what was right or wrong with the last iterations of the exercise and can more easily adopt his training. At the beginning, we only visualized the incoming data as described above. We soon added a judgment of the exercise quality per circle, but this was rated confusing by the users. Following completion of the training set an overall performance statement was given, providing motivation and overall feedback such as ' $30 \%$ of your exercises were correct.' This proved to be sufficient for the users. The coaches and physiotherapists get a per-circle judgement along with a per-circle visualization in their visualizing application. This different visualization for users and physiotherapist became possible after the inclusion of long-term data storage. This lead to a development of a specialized tool where the per-patient and per-exercise information of previously stored exercises is visualized. By using this tool, the physiotherapists and coaches are able to completely review all training sets within a minimum time and no direct supervision and annoyance for the trainee. By using their domainknowledge together with the sensor data, they can detect potential problems of the user, e.g. if he is not able to smoothly circle around due to reduced sinews in the legs or other potential problems. We found that their trained view at the visualization is also superior to any low-level interpretation logic. Therefore, no automated problem-recognition logic was added. But this could be a potential improvement for future system development. The initial versions were developed on a standard personal computer and demonstrated on a laptop. For real world deployment, we chose a touchscreen as this is easier to use while doing the exercises.

An easy-to-use graphical profile editor was developed allowing the physiotherapist to select users, define exercises for them and also to define new exercises. Along with the exercise parameters, a video can be added. This allows the user to review his next exercise. The exercises were conducted by an expert coach and videotaped. Users of the sports school already possess a RFID card which is used to authenticate them at the entrance of the sports school. To allow users to simply start their training, we added an RFID reader to our system. Now all necessary user data are retrieved at system logon. No further input is required. The user is also greeted by his name and given an overview of his current exercises. Currently, most people in the sports school carry their printed training plan with them as it is hard to remember the exercise parameters (weight, number of repetitions, etc.) for a large number of different machines. This is now included in our system. To allow data capture unrelated to specific exercises, which is important for the future research, we added a test mode where up to two therapy 
tops (at maximum one per foot) can be used for arbitrary exercises. The time from data capture to visualization is about 50 to $100 \mathrm{~ms}$. This small amount of delay is nearly unnoticeable, especially when really standing on a therapy top and performing the exercises. This allows for easy adaption and improvement in the exercise quality.

\section{Study and Evaluation}

We evaluated our system in a sports school with 47 participants over a two-weeks time frame. The participants completed 100 training sessions with our system. We had in total 21 female and 26 male participants, the youngest was 18 years old, the oldest 64 . The training sessions took part in a separate, private room within the sports school to prevent interruptions by other people. In the following, we concentrate on the results for the therapy top.

The participants performed one to four training sessions with our system. 42 participants were regular customers training at the sports school. Five were trainers who will be using the system later in their regular work. As part of their training plans, all participants had a number of exercises with one or two therapy tops. From their training plans we derived four sets of the most common exercise combinations. Those exercises were stored and associated to four RFID cards. Participants were already familiar with those cards as they are used for access control to the sports school. The 100 sessions took from 10 to 15 minutes each, depending on the training program and the rate at which the participants did the exercises. The training units with our system were done during the participants' regular training avtivity to ensure real training conditions. All raw sensor data gathered during the sessions was stored. We additionally videotaped a random selection of participants, resulting in 10 hours of video documentation. 32 of 47 participants returned the distributed questionnaires on time. We did not force an on-site return as participants stated that they wished to finish their regular training or did not have their glasses on in the sports school.

\subsection{Pre-Study Interviews}

For the evaluation, we interviewed the participants with a structured questionnaire to get background information, especially on their reasons for doing exercises with the therapy top and what problems they had with it. We were also interested in how many different exercises the participants were doing regularly and for what time period. We also asked them how they judged the correctness of the exercises they did and if they inquired for help if they were unsure or if they would like to be supervised at all or at certain intervals to improve their exercise quality.

The reasons for doing exercises with the therapy top were muscle build-up, improvement of the equilibrium sense, rehabilitation, injury prevention, improvement of the coordination capabilities and the motoric apparatus.

Common problems the participants stated to have were waggling over one side when tilting instead of tilting exactly over the middle, not completing whole circles during the circling exercises or not doing the exercises constantly. These issues can be addressed by our system. Problems we cannot address are skidding from the therapy top or moving the therapy tops apart while doing two therapy tops exercises. These problems are due to the nature of the exercises and the training equipment.

$36 \%$ of the participants stated that they already asked the trainers for a re-demonstration. They stated that this seemed necessary for them only when doing new exercises. Also, the problem to them was not understanding what to do, but to do it themselves. The minority $(27 \%)$ of the participants judged the correctness of their training as good or very good. This illustrates the difficulty of selfjudgment of the exercise quality. Nearly all of the participants stated, that they have already been corrected by a trainer more than 2 times. $92 \%$ of the participants stated that they always did not ask for support if they were unsure whether they were performing the exercises correctly. Also, most people would not like to have a trainer present when they were exercising. To them it was enough that trainers were available and making their rounds through the sports school. On these regular checks the trainers revise incorrect execution of exercises. Issues for not having a trainer present were 'I do not like to be watched.' or 'I know in principle how I should do it, but I do not get it right'.

After we explained the system to them, the participants were doing one of the four defined exercise sets. Each set of exercises comprised 3 different exercises each which had to be done twice with 30 repetitions of each time.

\subsection{User Study Accomplishment}

Participants were first demonstrated and taught the usage of the system:

- Authentication using an RFID card

- Reviewing the training plan (exercises, repetitions for each, amount of sets to complete) on screen

- Pressing the start button. For each exercise, a description of what to do and potentially a comment on how to achieve good performance was displayed. Before each exercise, there exists the possibility to view a movie of about 5 to 10 seconds where a trainer performs the current exercise. When people were ready, they placed the therapy top(s) in a convenient distance to the screen, potentially adjusting tilt and height of it. Then they mounted the therapy top and started exercising.

- While they were exercising, the user interface displayed a representation of the circle on the screen. 
- After finishing the repetitions for the exercises and completing the set of exercises twice, the application was returning to the authentication screen.

- Now participants were shown the visualization of their training, using a second application. Potential problems, e.g. waggling always over the left side in the tilt forward/backward exercise, were discussed with them. This could for example indicate a muscle disequilibrium in the legs. From the informal talk with them we were surprised that people stated that trainers previously discussed the very same issues with them when they were handed their training plans.

\section{$5.3 \quad$ Results}

We evaluated the system with the following key criteria in mind:

- Does the system give enough feedback?

- Can the users improve themselves with the system?

- Is the training more satisfactory to the user?

- Can training be done without any supervision?

- Is the user interface intuitive and usable?

- Do people become more aware of the errors they make?

The results we derived for these questions from our questionnaire are depicted in Fig. 1 and Fig. 2. We used a Likert scale with five options to assess these criteria. Depicted numbers above bars are the absolute number of people with respective opinion. Results show that all important issues were addressed properly. The system fulfils the users' expectations towards a computer-augmented therapy top system.
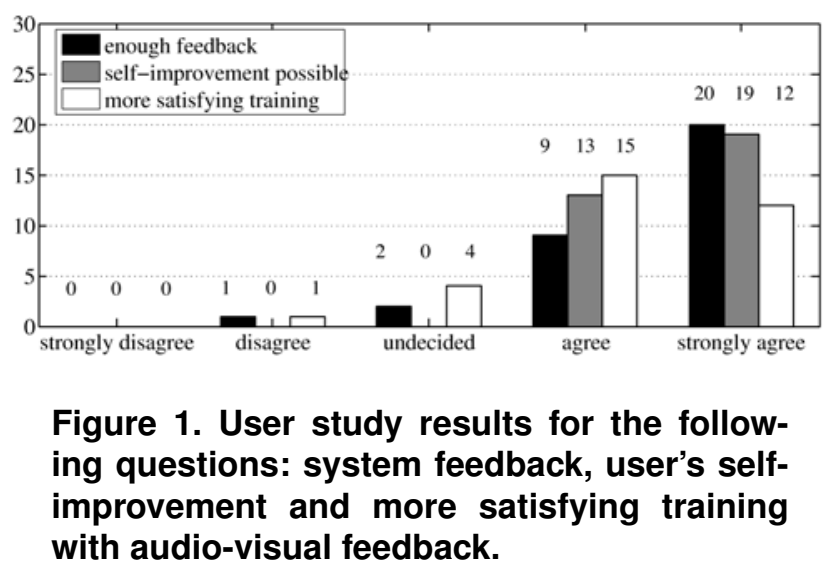

Participants stated that training with the audio-visual feedback of our system was increasing their motivation for doing therapy top exercises. This surprised us as people also stated that they in general disliked the therapy top exercises. The electronics and system components were rated as unobtrusive by all of the people. The electronics in the therapy top themselves is completely invisible and just signals operation by a blue LED underneath a cover.

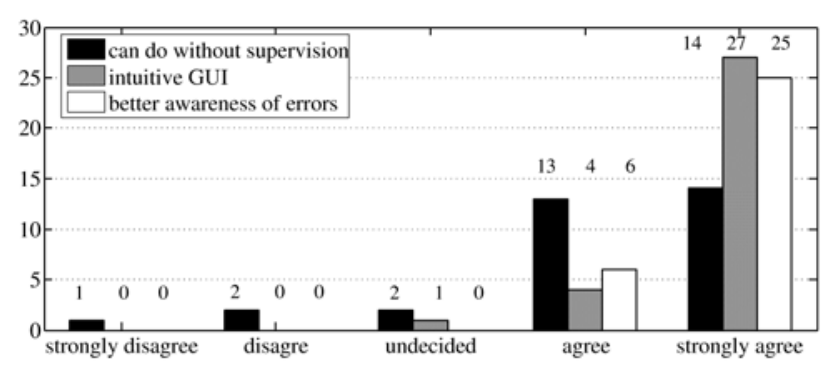

Figure 2. User study results for the following questions: training without supervision, usability of the GUI, error awareness.

We asked people if they would find a cable as appropriate. Most of them judged a cable as hindering and obtrusive. A cable would limit the possible placement of the therapy tops. Also, a cable would wind itself up during the circling exercises which would limit the usability in large parts.

The possibility of watching a pre-recorded video of a trainer doing the exercise was rated as only partially meaningful. The most often named reason by the participants was: 'I know what I am supposed to do and how but I simply do not get it right'. This was especially mentioned in conjunction with the first exercises for the people on the therapy tops and also with more advanced exercises.

We got interesting feedback from the trainers. They said that the possibility to define the tilt angles was a very good thing. Most people tend to just tilt back and forward very fast and not in a controlled manner. Defining tilt angles that are reasonable below the maximum tilt that can be accomplished with a certain therapy top could make people more aware of correct exercising if it forced them to stop the tilt movement at this angle.

We built the steel frame around our system to provide safety to our participants. In their normal training, they look for a free place nearby a training machine where they could get hold if something goes wrong, e.g. if they topple over. The dimension of the floor frame of $1.5 \mathrm{~m} \times 2 \mathrm{~m}$ was chosen as appropriately large. People stated that by visualizing the boundaries of the exercise place, they were more aware of incorrect exercising if they recognized that they needed more space.

\subsection{Improvements for the Therapy Top System}

Although the system was fairly well advanced and had no break down while in use, some issues still have to be addressed for further development. Some participants positioned two therapy tops mirrored compared to the visualization and did not notice it for a complete exercise. Also, going in wrong direction with the circling exercises was not 
always recognized by the participants, despite of the arrow in the visualized circle. This needs to be addressed, ideally the next version of the system would detect this and automatically give audio-visual feedback to the user.

Especially for the circling exercises, people tend to lift the heel as this makes circling at the back half of the exercise easier. A pressure sensor could help make people more aware of this problem.

Also, a gyrosensor instead of the accelerometer could supersede the correct placement of the therapy tops before the exercises, namely in a way facing towards the screen, as especially tilt exercises would otherwise be visualized in a wrong way with current system. Though, we did not mention the fact that the placement is critical, people did it correct nearly all the time. This is owed to the fact that people placed the therapy tops in a way they could read the number on the plate and an arrow on this plate was directing at the screen.

For a home scenario and to facilitate the data transmission as well as to pass the bandwidth limit, we are currently exploring switching to $\mathrm{WiFi}$ or Bluetooth data transmission. This will reduce the need for a specialized reception component, too.

\section{Conclusions}

We presented a user-centered design and development process for a ubiquitous computing system. We showed that highly interactive systems for end users in ubiquitous computing require different processes and tools during development. Especially fast iteration cycles and stakeholder integration are crucial. The processes were much more involving the users than with standard software engineering. We reported on the tools and steps we took to finally build a deployed and useful system in the field of sports. We highlighted how the specific steps can be generalized for the development of ubiquitous computing systems. We thereby hope to help researchers to build more deployed and working systems in this field of research.

\section{Acknowledgements}

The work has been conducted in the context of the research project Embedded Interaction ('Eingebettete Interaktion') and was funded by the DFG ('Deutsche Forschungsgemeinschaft').

\section{References}

[1] R. Asselin, G. Ortiz, J. Pui, A. Smailagic, and C. Kissling. Implementation and Evaluation of the Personal Wellness Coach. In IWSAWC, pages 429-535. IEEE Computer Society, June 2005.
[2] E. H. Chi. Introducing wearable force sensors in martial arts. Pervasive Computing Magazine, 04(3):47-53, 2005.

[3] E. H. Chi, G. Borriello, G. Hunt, and N. Davies. Sports technologies. IEEE Pervasive Computing, Special Issue On Mobile And Ubiquitous Systems, 2005.

[4] D. Fitton, K. Cheverst, M. Rouncefield, A. Dix, and A. Crabtree. Probing technology with technology probes. In Equator Workshop on Record and Replay Technologies, 2004.

[5] B. Friedman, P. Kahn, and A. Borning. Value sensitive design: Theory and methods. Technical Report 02-12-01, Comp. Sci. \& E., UW, Seattle, WA, USA, 2002.

[6] P. Holleis, M. Kranz, A. Winter, and A. Schmidt. Playing with the real world. PerGames 2005, May 2005.

[7] P. Holleis, M. Kranz, A. Winter, and A. Schmidt. Playing with the Real World. Journal of Virtual Reality and Broadcasting, 3(1), Apr. 2006.

[8] H. Hutchinson, W. E. Mackay, B. Westerlund, B. B. Bederson, A. Druin, C. Plaisant, M. Beaudouin-Lafon, S. Conversy, H. Evans, H. Hansen, N. Roussel, and B. Eiderbäck. Technology probes: inspiring design for and with families. In G. Cockton and P. Korhonen, editors, CHI 2003, pages 17-24. ACM, 2003.

[9] J. A. Kientz, S. Boring, G. D. Abowd, and G. R. Hayes. Abaris: Evaluating automated capture applied to structured autism interventions. In Ubicomp '05, pages 323-339, 2005.

[10] J. C. Kimel. Thera-network: A wearable computing network to motivate exercise in patients undergoing physical therapy. In ICDCS Workshops, pages 491-495, 2005.

[11] S. R. Klemmer, J. Li, J. Lin, and J. A. Landay. Papier-mâché: toolkit support for tangible input. In $\mathrm{CHI}$ '04, pages 399406, New York, USA, 2004. ACM Press.

[12] M. Kranz, P. Holleis, W. Spiessl, and A. Schmidt. The Therapy Top Measurement and Visualization System - An Example for the Advancements in Existing Sports Equipments. In Sport und Informatik IX. Proceedings of the 6th Workshop of the dvs-Section Computer Science in Sport, pages 201-210. Shaker, June 2006.

[13] M. Kranz and A. Schmidt. Restriction, modification and extension of consumer devices for prototyping ubiquitous computing environments. In ICDCSW' 06 , page 57, Washington, DC, USA, 2006. IEEE Computer Society.

[14] F. Michahelles and B. Schiele. Sensing and Monitoring Professional Skiing Athletes: Lessons Learned from a Collaboration with Ski Trainer's. Pervasive Computing Magazine, 03, July-September 2005.

[15] A. Page, A. Cooper, and D. Sutch. Futurelab Fizzees. http://www.futurelab.org.uk/showcase/ fizzees/. visited January 2006, January 2006.

[16] Research Group Embedded Interaction. UbiFitness Project Page. http://www.hcilab.org/projects/ ubifitness /, visited June 2006, June 2006.

[17] A. Schmidt, P. Holleis, and M. Kranz. Sensor virrig - a balance cushion as controller. Workshop Playing with sensors at UbiComp 2004, September 2004.

[18] J. Scholtz and S. Consolvo. Towards a discipline for evaluating ubiquitous computing applications. Technical Report Intel Research, No. IR-TR-2004-25, February 2004.

[19] H. Sharp, A. Finkelstein, and G. Galal. Stakeholder identification in the requirements engineering process. In DEXA '99, page 387, Washington, USA, 1999. IEEE. 